\title{
El trabajo infantil en la zona rural con edades de escolaridad
}

\section{Child labor in rural areas with schooling ages}

\author{
Avendaño, Juan de Dios; Editor Academico Dr. Angel Sol Sanchez
}

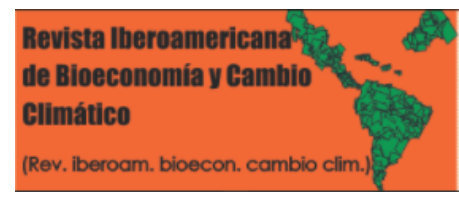

Juan de Dios Avendaño

Universidad Nacional Autónoma de Nicaragua- León. UNAN-LEÓN, CUR Somotillo, Nicaragua

Editor Academico Dr. Angel Sol Sanchez

Colegio de POsgraduados Mexico, México

Revista Iberoamericana de Bioeconomía y Cambio Climático

Universidad Nacional Autónoma de Nicaragua, León, Nicaragua ISSN-e: 2410-7980

Periodicidad: Semestral

vol. 2, núm. 1, 2016

czuniga@ct.unanleon.edu.ni

Recepción: 30 Agosto 2015

Aprobación: 24 Abril 2016

URL: http://portal.amelica.org/ameli/journal/394/3941750023/

DOI: https://doi.org/10.5377/ribcc.v2i1.5701
Resumen: El estudio se centró en discutir el tema del trabajo infantil desde una perspectiva orientada en el sujeto; un tema altamente controvertido y marcado por las diversas emociones. El punto central son los propios niños trabajadores. Se analiza su trabajo primordialmente bajo el aspecto de los significados que tiene para ellos mismos. De hecho a pesar de muchos trabajos que aborda sobre los derechos del niño es frecuente ver en la televisión y en la prensa imágenes y artículos de niños trabajadores de países en desarrollo, mal alimentados y pobremente vestidos. En los albores del nuevo milenio, el trabajo que realizan estos desafortunados niños es un rasgo inaceptable de la vida de muchísimos países. La secuelas que más se ven son las edades de nuestra niñez quecorresponden para estar en los primeros grados de los centro escolares rurales y que muchas veces vemos que algunos padres le pone menor importancia a esa etapa ya que es muy fácil de manejar. A fin de que ellos logren terminar sus estudios y cuando lo termina lo hace con poca destreza y asimilación de contenidos básicos. Simultáneamente, algunos docente quiere ser parte de la solución a este flagelo; aunque la evaluación no son aplicada profesionalmente y esto incluyen que los niños prefieren trabajar que estudiar por lo que resulta una formación a largo plazo y con una dedicación muy esmerada por los actores que conforma la educación: padres, alumnos y docentes; sin poner fuera de contexto la ayuda de los gobierno.

Palabras clave: Enseñanza, Huerta, Ayuda, Carencia, Escolaridad.

Abstract: The study focused on discussing the issue of child labor from a subject-oriented perspective; a highly controversial subject marked by various emotions. The central point is the working children themselves. Their work is analyzed primarily under the aspect of the meanings they have for themselves. In fact, despite many works that address the rights of the child, it is common to see on television and in the press images and articles of working children from developing countries, poorly fed and poorly dressed. At the dawn of the new millennium, the work of these unfortunate children is an unacceptable feature of life in far too many countries. The sequels that are seen the most are the ages of our childhood that correspond to being in the first grades of rural schools and that we often see that some parents give less importance to that stage since it is very easy to manage. In order for them to finish their studies and when they finish it, they do so with little skill and assimilation of basic contents. Simultaneously, some teachers want to be part of the solution to this scourge; although the evaluation is not applied professionally and this includes that children prefer to 
work than study, which results in long-term training and with a very careful dedication by the actors that make up education: parents, students and teachers; without putting government aid out of context.

Keywords: Teaching, Vegetable plot, Help Lack, Escolary.

\section{INTRODUCCIÓN}

A pesar de que el trabajo infantil es un concepto ambiguo, difícil de definir. En el caso específico de las zonas rurales, la incorporación gradual de los niños al trabajo es aceptada como un hecho. La naturaleza de dicha incorporación difiere de sociedad en sociedad y tiene significados diversos, tanto para los niños como para sus familias.

En la mayoría de los casos, el problema encuentra sus raíces en las situaciones de pobreza vivida por segmentos importantes de la población rural. Entre sus causas fundamentales, destacan el desempleo y subempleo, los ingresos precarios, y en general, la desigual distribución de bienes y beneficios sociales que afectan la calidad de vida en el sector.

Por lo que sea ha realizado una reflexión en la observación educativa con diferentes enfoques como: investigación en el aula, investigaciones colaborativa, investigación participativa, investigac ión crítica, etc., que designan resultados investigativos con cierta especificidad, pero que se consideran expresiones frecuentemente útiles y destacadas como el resultado de lo que se busca

Además esto nos ayuda a interpretar elementos colaborativos encontrados en diferentes fuentes de referencia que nos ayuda a interpretar el desarrollo curricular, su autodesarrollo profesional, la mejora de los programas educativos, los sistemas de planificación o la política de desarrollo. Estas actividades tienen en común la identificación de estrategias de observación que son implementadas y más tarde sometidas a acción reflectada.

Desarrollo

"La educación es una actividad sobre la que todo el mundo tiene alguna experiencia, como alumno, como padre o como profesor. Así, ocurre que todo el mundo opina sobre ella; mientras que sobre física o genética no se atreven a hablar más que los especialistas, sobre educación habla cualquiera, y lo que es peor, toman decisiones personas que piensan que pueden basarse en sus experiencias de la vida cotidiana sin una mayor visión del conjunto del sistema y sin aplicar los esquemas científicos y técnicos que reclaman para el resto de las decisiones públicas" corrobora Esteve, J. M. (2003); esta primera cita emblemática nos da pautas para reflexionar sobre la brecha existente entre el diseño de las políticas educativas y su ejecución en el aula en zonas rurales.

Es necesario reincorporar a los niños que desertan por motivos económicos, y en particular por la ineficacia de la escuela, para vincularse al mercado de trabajo. Por lo tanto, exhorta a impulsar un proceso de apropiación colectiva del sistema escolar para convertirlo en un ámbito público, y desarrollar la capacidad social de plantear problemas y soluciones al generar responsabilidades compartidas en los autores principales de la educación en la sociedad.

Debido a esta situación son más los varones que no se matriculan por razones de trabajo. Las jóvenes mayores de 15 años no se matricularon por tener que desempeñar los oficios domésticos.

Luego que Garrabou, R. (2000) reafirma que: "Estos si presenta problemas entre propiedad y explotación campesina y la naturaleza del trabajo agrario."

No obstante, hay una particularidad en zonas rurales que provoca un quiebre entre el calendario agrícola y el calendario escolar porque esto provoca el abandono temporal y, en consecuencia, el atraso de estos educandos. 
Según Arenas Monreal, et. al. (1999), "la precaria situación nutricional de la población infantil en comunidades campesinas e indígenas, nos obliga a reflexionar sobre los factores socioeconómicos que la determinan:"

Por las actividades laborales que desempeñan durante largas jornadas, estos niños llegan tarde a las aulas hambrientos; en otros casos llegan cansados y pierden la concentración, o no tienen tiempo para estudiar y hacer las tareas en casa. En pocas palabras, trabajar significa una carga que daña un probable mejor desempeño en la escuela. La forma como el trabajo afecta el rendimiento en las aulas está asociada a la extensión de la jornada laboral, edad y tipo de actividad.

Como Briceño Ayala, L., \& Pinzón Rondón, Á. M. (2004), identifico que: “Las causas del trabajo infantil son múltiples y entre estas podemos destacar: la pobreza, que constituye un círculo vicioso al asociarse con el trabajo infantil, la creencia errada que hace pensar que el trabajo infantil es bueno y la carencia de políticas socioeconómicas en contra del trabajo infantil”.

Por supuesto, muchas familias de la zona rural piensan que la educación es para los ricos, familias profesionales que contenga el nivel económico propicio para que sus hijos estudien y sea buenos profesionales. Por lo tanto, estudiar para estas familias es como un paseo a Disney Landia difícil, absurdo y complicado para sus recurso económicos que muchas veces ellos siente que con muchas dificultad logran conseguir los alimentos mínimo y balanceados que una familia común puede conseguir.

Prontamente que Salazar, M. C. (1996), afirma que "Los aportes económicos de estos niños al presupuesto familiar no son muy significativos: se calcula que suman alrededor de un $10 \%$ de ese presupuesto. Es tres veces mayor el aporte de los adolescentes (de 13 años en adelante) al presupuesto familiar, y ellos predominan dentro de la fuerza de trabajo de los menores de edad. A medida que transcurre la edad, un contingente mayor se incorpora al trabajo, siendo así que la mayor proporción de trabajadores menores de edad en nuestros países está localizada en el grupo de 15 a 17 años."

Inicialmente, se debe vencer este problema en muchos padres de familias en zonas rurales ven al centro escolar como un lugar poco beneficioso y suculento para alcanzar los recursos necesarios que todo hogar debe tener a la cual el estudio constituye un gasto más y que sus finanzas se ven en riesgo. No podemos omitir que los ingresos obtenidos por los padres en su trabajo así será el nivel de vida familiar en el campo.

Rápidamente que Salazar, M. C. (1996) certifica que"Muchos padres temen a la escuela y al estudio. "Yo aprendí así", es común que lo digan. Recoger su propia experiencia anterior como niños trabajadores para justificar el trabajo de sus hijos que es uno de los mecanismos más utilizados.” (Garrabou R, 2000)

Si bien es cierto, que meter en la cabeza a un niño el amor por el trabajo no es malo, tampoco lo es integrarlo que ayude en las actividades del hogar, lo malo es aprovecharse de la necesidad y condición de pobreza de los menores de edad y su inocencia como lo hacen algunos adultos que tiene su parcelas que hasta sus mismos padres que muchas veces se aprovechan, ven en el hijo un medio para obtener dinero obligándolos a realizar "pequeños" trabajos interrumpiendo de esta manera su vida escolar.

Inmediatamente que Salazar, M. C. (1996) atestigua que "Los padres justifican la vinculación de sus hijos al trabajo aduciendo que en él adquieren valores como la responsabilidad, la autonomía y la tenacidad para sobrellevarlas dificultades o para soportar sacrificios. Además se interpreta al trabajo como una protección contra los vicios y el ocio "que conduce a la delincuencia".

Esta aseveración demuestra que cuando nos sumergimos en la realidad de una comunidad rural, en las en las entrañas culturales del campesinado, descubrimos el mundo real de la mayoría de las comunidades de escaso recursos económico. Hay un escenario oculto pero latente, del cual mucho se discute y poco se toma en cuenta que es la pobreza extrema con una calidad de vida no deseable.Además los padres reafirma que teniendo a sus hijos trabajando los alejas de toda mala influencias que los puedan contaminar; otros dicen "cuerpo ocupado mente bloqueada."

En seguida que Sapelli Claudio (2004), establece que "Para ello presenta un modelo micro econométrico de elección bivariada que permite estudiar conjuntamente dos decisiones diferentes, pero relacionadas: la de 
deserción escolar y la de participar en la fuerza laboral. Estas decisiones pueden considerarse relacionadas ya que muchos de los que desertan lo hacen para trabajar, pero son diferentes, puesto que no todos los jóvenes que desertan van al mercado laboral, algunos permanecen en sus hogares, o trabajan en la familia y no son remunerados."

Efectivamente, se refieren a aquellas familias que viven en un espacio artesanal y poco salubre, en donde el padre de familia -si es que existe- trabaja de sol a sol en tareas hostiles y precarias, mientras la madre de familia vive acarreando agua y leña la mayor parte de su vida, y quienes tienen parcela o animales de granjas; los niños y niñas trabajan a temprana edad para apoyar a su familia donde realizan diferentes tarea propias del bien común familiar. Sin omitir que cuando ellos obtienen algo de dinero se los da a sus padres todos ellas, pero cuando el padre consigue su propio dinero da una parte y la otra la comparte en la cantina con los amigos sin importar si lo que dio es suficiente para la manutención de sus hijos.

Al punto que Khoudour Castéras, D. (2007), menciono que: "En general, existe mayor riesgo vulnerabilidad frente a la explotación laboral y la trata infantil cuando niños, niñas y adolescentes migran de manera autónoma."

En cual se emplean una gran cantidad de mano de obra infantil en otros lugares o países, siendo esta más barata, eficiente y de fácil manejo no teniendo espacios para jugar y aprender, y en pocos casos, los adultos no asumen sus responsabilidades de padres protectores y proveedores de las necesidades del infante. En la cual ellos prefieren que sus hijos trabajen con sus amigos bajo el concepto de que deben aprender un oficio como: albañil (más bien ayudante de albañil)

Además que Ávila A. S (2007,) aseveró que "El trabajo infantil no sólo es ilegal, moralmente inaceptable y un ultraje a la dignidad humana. No sólo lastima a los niños y niñas que por derecho deberían estar estudiando en lugar de trabajar, sino que además resulta poco rentable en términos económicos".

Igualmente Sapelli Claudio (2004) establece que "Para ello presenta un modelo micro econométrico de elección bivariada que permite estudiar conjuntamente dos decisiones diferentes, pero relacionadas: la de deserción escolar y la de participar en la fuerza laboral. Estas decisiones pueden considerarse relacionadas ya que muchos de los que desertan lo hacen para trabajar, pero son diferentes, puesto que no todos los jóvenes que desertan van al mercado laboral, algunos permanecen en sus hogares, o trabajan en la familia y no son remunerados."

Esto es un aspecto muy frecuente ya que los padres quienes poseen una parcela o ciertos numero de ganado trabajan duro; para conservar el fruto de su trabajo de una vida y poder entregárselos a sus hijos promoviendo el patrimonio familiar como una herencia compartida y trabajada; donde ellos para poder obtener algo de ella deben ocuparse con mucho esmero en su área de cultivo o al cuido de sus animales de granjas que han acumulado durante su periodo de vida humana.

$\mathrm{Al}$ instante que Klein, Emilio (1993), asegura "La mayoría de ellos pertenece a familias que viven de la agricultura, una parte significativa son pobres y es muy probable que en la próxima década una proporción importante de ellos ya no estén más allí: se habrán ido a las ciudades, al igual que millones de sus coterráneos que lo han venido haciendo desde hace medio siglo."

No obstante, en la calidad de vida de los estudiantes de zona rural, de cada diez niños y niñas que asisten a la escuela, siete trabajan en oficios domésticos o en tareas agrícolas. Estos niños no juegan, viven una prematura y forzosa pre - adolescencia. Tienden a fracasar y a abandonar la escuela, y por su escasa educación tendrán menos oportunidades de desarrollo, y un pronóstico reservado en términos de promiscuidad sexual, pero con mayor índice de participación en grupos de pandillas o emigración.

Posteriormente que Salazar, M. C. (1996), tienen acentuada presencia, en particular en zonas rurales, concepciones pre -modernas de la infancia, en las que niños y niñas son concebidos solamente como "adultos en miniatura”. En estos casos los niños son recargados de actividades laborales, en tanto éstas forman parte de su función como tales". 
Es por ello que el trabajo infantil es un fenómeno multidimensional del que es delicado aislar sus elementos sociales, culturales y económicos, pues en cada país o región está estrictamente vinculado entre sí. Por lo que son muy diversas y difícil de separar estos factores lo que están determinados por una variación de situaciones, actitudes y valores que pueden predisponer a la familia y comunidad ver el trabajo de infantes como algo nato, fomentándolo y aceptándolo.

Por consiguiente que Salazar María Cristina (1996), relaciona que "entre la escuela y el trabajo infantil varía según se dé en regiones urbanas o rurales, y de acuerdo a contextos Socioeconómicos y geográficos, lo mismo que según tipos de familias. En sectores rurales, los estudios indican que prácticamente todos los niños, varones y hembras, participan del trabajo doméstico y todos también, desde temprana edad, llevan a cabo actividades relacionadas con la agricultura, sea que asistan o no a la escuela".

Aparte de lo antes mencionado, en este contexto se puede identificar las bajas tasas de cobertura en zona rurales y la finalización de la educación secundaria de pocos estudiantes que terminaron su ciclo escolar de primaria y no continúan la secundaria porque ellos viven en zonas muy retiradas donde los centros de secundarias están lejano y no hay medios de transportes en que trasladarse. Por ende; sus deseos de continuar sus estudios se ven mutiladas y con un porvenir estudiantil poco probable para alcanzar una profesionalización educativa en una etapa técnica o universitaria.

Ávila A. S (2007), estableció que "La deserción escolar en educación básica y educación media superior, es mayor en los hombres que en las mujeres. La diferencia entre hombres y mujeres que abandonan la escuela se incrementa conforme se avanza en los niveles escolares."

Aunque, esta analogía no se ve como una lógica matemática proporcional positiva al contexto real de nuestra población rural. La falta de cobertura es una limitante que están asociados a la inequidad, ya que con frecuencia los niños, niñas, adolescentes o jóvenes que no pueden continuar estudiando en un grado subsiguiente o nivel superior tendrán como trascendencia una decadencia en su calidad de vida y educativa por la distancia en donde viven.

Por lo tanto que Alarcón Glasinovich, (2004) estableció que "En algunos contextos, especialmente los pobres, el trabajo es percibido por muchos padres y madres como una suerte de escuela para la vida, como una forma de capacitar a sus hijos no sólo en términos de alguna habilidad o conocimiento, sino para enseñarles lo que es la vida."

De igual forma, por lo que muchos padres espera en ellos que se mantenga más tiempo en el campo; menos en su escuela, ya que es única base de educación en la que está ahí y sus docentes son los mismo padres que enseña empíricamente y con rudeza; los conocimientos y experiencias de la vida transmitida de generación a generación.

Más tarde que Según Salazar María Cristina (1996);” los estudios se refieren a la situación atrasada, muchas veces desastrosa de la educación; se habla de la "escuela fracasada" que en lugar de atraer a los niños y niñas, los expulsa. Se señala la urgencia de transmutar el sistema educativo, crecer la inversión estatal en educación, mejorar los niveles de los maestros e introducir pedagogías nuevas, para que la escuela se convierta en imán de los niños. Se destaca la necesidad de una voluntad política en la que tiene como finalidad de retener a los niños en la escuela como el primer objetivo por lograr dentro de la meta de erradicación del trabajo infantil."

La política educativa en Nicaragua con respecto a la retención escolar y la calidad de la educación cuenta con una dinamización de la calidad de los aprendizajes en los alumnos. Por lo siguiente se estableció un análisis sobre los aprendizajes en la zona educativa en la qu resulto como de baja calidad en lo que argumentan que muchas veces los niños y niñas no tiene el suficiente tiempo para realizar sus tareas ya sea teóricos, practico e incluso investigativo. Por ende uno de los principales problemas es el absentismo o inasistencia en las clases por que la calidad de aprendizajes es muy reducida y muchas veces nulas.

Al mismo tiempo; la evaluación de los aprendizajes y en desarrollo de los contenidos son otro fenómeno que está ocurriendo en los centros educativos que minimizan al estudiante donde ellos ve al centro de estudio 
como una recamara de terror y no como un lugar agradable que le permite demonstrar sus capacidades, aptitudes o actitudes a los diversos saberes propuesto por el gobierno.

"y porque no me siento orgullosa de ganar algo yo solo, aprendo a manejar dinero y soy independiente. Si no ganamos dinero, no podemos curarnos ni ponernos lo diente ni comer. Si no trabajamos estaríamos viviendo en una crisis económica aun peor que la que estamos viviendo ahora. Nos gusta estar con otras personas y podemos jugar y ayudar a otros." (Liebel, Manfred, 1996)

En la zona rural los niños prefieren ir a trabajar al campo que estudiar porque siente que sus condiciones intelectuales son muy indiferentes a aquellos niños o niñas que no tienen que levantarse muy de mañana a realizar labores de campos antes de ir al colegio Por ende; entre estudiar y trabajar prefieren trabajar por lo que más fácil, práctico y lucrativo que estudiar que conlleva un esfuerzo, tiempo y dedicación para lograr las metas educativas propuestas por el sistema.

Seguidamente que Llop, J. M. B. (2000) afirma que: hay datos sobre matrícula y asistencia escolar, puso de relieve, entre otras cuestiones, el alcance de un absentismo crónico muy extendido, " uno de los más graves agujeros negros de la realidad educativa del siglo XIX y comienzos del XX"

Puesto que Briceño Ayala (2004), enfatizo que: "más de 246 millones de niños entre los 5 y los 17 años se encuentran actualmente trabajando en todo el mundo y 180 millones se encuentran sometidos a las peores formas de trabajo infantil, como lo son la explotación sexual, las tareas domésticas, el trabajo en minas, en fábricas de fuegos pirotécnicos y en los conflictos armados."

Aparte de que algunos actores menos precia el coeficiente del niño o niña en la escuela debido a que otros ven como su capacidad laboral en el campo es valorado positivamente con sus actitudes y habilidades expresando por tercera personas: este niño es fuerte, mira como monta el caballo, ves él o ella sabe enrejar una vaca y la puede ordeñar sin ayuda, se está convirtiendo en un varoncito, ella es muy aseada y sabe cocinar muy bien, etc.

"La infancia es una etapa de desarrollo rápido, y en ella adquirimos las aptitudes y conocimientos que nos permitirán ser adultos productivos y buenos ciudadanos. El aprendizaje y la adquisición de aptitudes se realizan tanto mediante la enseñanza escolar (leer y escribir, aritmética...) como mediante la experiencia del trabajo y de la vida (autonomía, responsabilidad, aptitudes y conocimientos tradicionales...).(Liebel, Manfred, 1996)

Al mismo tiempo, si el maestro o maestra no sabe dirigir o en caminar los múltiples ritmos de aprendizajes en niños y niñas quienes hacen labores en el campo o no; a la cual estudian en el mismo centro y algunos no tiene que madrugar para ir al colegio; por lo que induce a la frustración cuando aprenden rápido y luego se ponen a jugar, ante lo cual el docente los castiga, ignorando que esa actitud es por simple aburrimiento que ha generado el propio maestro o maestra. En contra parte del otro alumno que tiene problemas de aprendizaje, es lento para adquirirlos y se tiene que levantar de madrugada en su hogar se ve en si mismo como alguien poco capaz de igualarse a sus compañeros de clases.

Conjuntamente, Según Anker, R. (2000), argumenta que "Sin embargo, tampoco podemos afirmar tajantemente que todas las formas de trabajo infantil sean un obstáculo inevitable para la asistencia a las clases $y$ el rendimiento escolar. Aunque el trabajo a tiempo completo (sea peligroso o no) es obviamente incompatible con la vida escolar, no ocurre lo mismo con la actividad a tiempo parcial si ésta se realiza durante las vacaciones escolares o durante unas cuantas horas a la semana a lo largo del curso. Aunque no sabemos con certeza cuánto tiempo puede trabajar un niño sin que ello perjudique a su rendimiento escolar, parece verosimil que sea de por lo menos dos o tres horas diarias.

Tomando en cuenta lo anterior y todo el proceso educativo donde el estudiante obtiene una nota reprobable es uno de los errores más graves de los docentes, de los estudiantes y de los padres y madres de familia calificando a sus hijos o hijas de la zona rural poco hábil para alcanzar un aprendizaje integral, significativo y permanente. Por ende, se les inculca que es mejor trabajar que estudiar ya que estudiar es complicado y frustrante, por lo general el niño o niña al verse desilusio nado cree en una verdad a medias. 
Próximamente que Stewart, M. A(2007), descubre que: "Vivir en la calle es como es como pelear por tu vida, si no sobrevivís es como una guerra fría diaria, es ciertos que la vida en la calle es muy dura y muy pesada. Pero para personas que no han vivido en las calles que siempre han tenido las cosas necesarias para vivir bien y cómodamente, es difícil entender verdaderamente esta vida que es la realidad para numerosos personas.”

Aparte de que es habitual ver por las calles, paradas de autobuses, mercados a niños y niñas vendedores de agua, refrescos y golosinas sirviendo como intermediarios entre el dueño de un negocio, quien les garantiza treinta o cincuenta córdobas por un día de trabajo que normalme nte empieza desde las cuatro de la mañana y termina a las diez de la noche, donde muchas veces se vuelve más consciente de su lugar dentro de la familia siendo un pilar más dentro de esta economía sin omitir que quizás sean ellos adolescentes que toma el rol progenitor ya que sus padres quizás algunos sea un alcohólico, drogadicto o irresponsable en el papel de padres que deben cuidar a su prole

"Para contrarrestar la falta total de información sobre las niñas escolarizadas, se ha recurrido al Padrón Municipal, con el fin también de contrastar y completar los datos sobre niños escolarizados y sus familias. Finalmente, si se quiere obtener además información sobre los tipos de tareas laborales ejecutadas por niños y niñas es imprescindible recurrir al testimonio oral de los protagonistas."

Esto ha permitido tener un control exacto de la población estudiantil que están estudiante y a la vez permite ver que el presupuesto del 'país está siendo bien administrado ya que los primeros beneficiado en este rubro son la niñez.

De modo que Sapelli Claudio (2004), establece que "Cuando la deserción se asocia a la incorporación a la fuerza de trabajo, esta decisión presenta beneficios inmediatos por el dinero ganado en el trabajo y beneficios de mediano plazo, que surgen de la más temprana experiencia laboral; pero también presenta efectos negativos de mediano y largo plazo, que surgen de un abandono temprano de la enseñanza formal, en términos de menor capital humano y posiblemente menores ingresos en una perspectiva más permanente."

Además, que Ferrer, A. T. (2010), estableció que "Entre la fecha de aprobación de tales leyes y la de su aplicación efectiva hubieron de transcurrir lapsos de tiempo más o menos dilatados, según los países. En tales períodos, la escolarización de los hijos de ciertos sectores sociales coexistía con la inasistencia escolar de otros muchos niños. Entre los factores que contribuirían a explicar tal estado de cosas habría que incluir la escasez de plazas escolares, los problemas presupuestarios de algunos países, el poco aprecio de las clases populares por la educación, pero también, y es asunto que interesa aquí especialmente, el mantenimiento de un mercado laboral infantil que convenía al sistema productivo."

Conjuntamente de que se relata experiencias de adolescentes migrantes en el mundo y casos de jóvenes víctimas de trata de personas, en particular de explotación sexual (prostitución infantil) y laboral (trabajo doméstico).Los investigadores han centrado su interés en la relación entre remesas, educación y trabajo infantil, donde los gobiernos no ponen atención a este problema, como subrayan Akabayashi y Psacharopoulos (1999), existe una relación inversa entre educación y trabajo infantil. De hecho, el tiempo que los niños y las niñas dedican al trabajo representa un costo de oportunidad que va en detrimento de sus estudios

Por lo tanto que Ferrer, A. T. (2010) fundó que "Legalmente que los gobiernos proclamó que la educación debía ser universal, pública y gratuita, El trabajo infantil convenía por igual a empresarios y obreros. Para los patronos, los bajos salarios de los niños (y las mujeres) permitían paliar la escasa productividad y falta de competitividad de muchas industrias. Para las familias obreras, el salario de los niños era un complemento indispensable para el presupuesto familiar, en una época de carestía y alto coste de la vida7. En consecuencia, existieron unas condiciones favorables al mantenimiento del trabajo infantil, y desfavorables para el logro de la escolarización universal."

Cuanto más dinero necesitan las familias, menos tiempo disponible hay para la escuela. Además, la educación, aun cuando es gratuita, tiene un costo en la medida en que significa gastos suplementa $\mathrm{r}$ ios para las familias, en particular en útiles escolares, uniformes y transporte. 
También que Ávila-Curie (1998) dice que: "La muchos de los niños que trabaja en la zona rurales están desnutrido por falta de alimentos nutritivos que pocas veces los padres consigue en su trabajo."

Los niños se encuentran en una etapa del desarrollo tanto físico como psicológico muy importante y tanto fisiológica como psicológicamente son inmaduros, por esta razón son mucho más vulnerables que los adultos a la exposición a cualquier factor de riesgo. Teniendo en cuenta que los ambientes laborales presentan una gran cantidad de factores de riesgo, los niños en lo posible no deben ser expuestos a estos ambientes. La salud es un derecho de los niños y se debe propender por su promoción y por la prevención de enfermedades.

Desafortunadamente este es una realidad muy sentida en las zonas rurales ya que en el ámbito legal no existe un límite claro entre lo permitido y lo prohibido en trabajo infantil que tiene como excusa el hambre en el hogar. Casi todas las personas han tenido algún tipo de trabajo antes de cumplir los 18 años y esta experiencia puede traer cosas positivas cuando el éxito toca a su puerta. Sin embargo, existe hoy en el mundo una gran cantidad de menores que ven truncado su futuro por comenzar a trabajar prematuramente.

No obstante, Maureira Estrada Fernando (2000) asegura que: "Estas dos perspectivas complementarias, la primera horizontal (reproducción de la fuerza de trabajo) y la segunda vertical (desarrollo de los sectores productivos capitalistas y de la economia informal), nos permitirán esclarecer las formas que adopta el trabajo infantil como parte de una estrategia más amplia de sobrevivencia y de reproducción social."

Diferentes investigaciones científicas han demostrado como el trabajo afecta el desarrollo de los niños y jóvenes, los expone a sufrir accidentes y los predispone a desarrollar múltiples enfermedades. Los niños son más vulnerables que los adultos por la etapa de desarrollo en la cual se encuentran. Además algunos niños y jóvenes en el trabajo se encuentran bajo presión porque desconocen el ambiente laboral, necesitan conservar su trabajo y deben aportar al sustento diario de su familia. Los niños son tan vulnerables a los factores de riesgo laborales que incluso algunas investigaciones han mostrado como la exposición laboral de los padres influye en su salud al afectarlos en etapas muy tempranas de desarrollo.

En cuanto al desarrollo y la madurez psicosocial, los niños se encuentran en proceso de evolución de su personalidad y por esto trabajos con peligro de violencia, abuso o adicción los predisponen, aún más que los adultos, a sufrir trastornos psicosociales y además los hace más vulnerables a situacio nes laborales con alta exigencia mental y física.

Por eso, el niño es el gestor de su propio desarrollo y son muy importantes su autoestima el desarrollo de su creatividad, y su felicidad. Los niños y niñas trabajadoras con frecuencia trabajan en actividades que son explotadoras, peligrosas y degradantes. Muy a menudo son maltratados, abusados y abandonados por sus patrones. Estos niños están especialmente propensos a sufrir alteraciones psicosociales. (Latorre Antonio, 2003)

Además el trabajo infantil interfiere con el desarrollo social y académico de los niños. El trabajo les quita tiempo para desarrollar otras actividades como jugar, aprender y compartir en familia. Varias investigaciones han mostrado como los niños trabajadores tienen una muy alta deserción escolar y un alto retraso escolar

Las interacciones negativas entre las condiciones de trabajo y los factores humanos pueden conducir a perturbaciones emocionales, problemas de comportamiento y cambios bioquímicos y neutro-hormonales que presentan riesgos adicionales de enfermedades mentales y físicas. Pueden también preverse efectos nocivos para la satisfacción y el rendimiento en el trabajo.

Accidentes. Los niños y jóvenes son nuevos en el trabajo, inexpertos, no se atreven a preguntar sobre su trabajo ni a hacer exigencias y no conocen sus derechos como trabajadores, esto los hace especialmente vulnerables a sufrir accidentes en el trabajo. (Gattino Silva; Nora Aquino, 1999)

Enfermedades Ocupacionales. Algunas enfermedades que se han visto relacionadas con exposición a factores laborales son:

Asma: La aparición de asma a temprana edad ha sido asociada a humo de madera, petróleo, carbón, exhostos, herbicidas, pesticidas, polvo de granjas, granjas con cosechas y granjas de animales. 
Cáncer Infantil: La leucemia linfoblástica aguda y el cáncer cerebral son lesiones que típicamente se han encontrado más en niños expuestos a agentes tóxicos. (Gajardo M; Andraca A M, 1988)

Desórdenes del Neuro-desarrollo: De profunda incidencia en su vida futura, se ha detectado el desarrollo de discapacidades del aprendizaje, dislexia, retardo mental, desorden de déficit de la atención y autismo.

En la actualidad se estudian los efectos neuro-psicológicos de los contaminantes y por tanto se determina el estado de variables como la atención y la concentración, la función sensorial, el control motor, las relaciones espaciales, la coordinación viso motora, la memoria y el afecto. (Esteve J M, 2003)

Asimismo que Ray, R., \& Lancaster, G. (2005), definió que: "Los coeficientes de la variable «años de escolaridad» indican que la asistencia a la escuela puede «crear costumbre» en el sentido de que, ceterisparibus, cuanto más tiempo ha pasado un niño en la escuela, menos peligro corre de abandonar los estudios. Si el hogar dispone de agua y luz eléctrica y posee bienes como televisión y teléfono, hay más posibilidades de que el niño sólo se dedique a estudiar y no comience a trabajar. "

Igualmente Ávila, A. S. (2007) reafirma que: “Si bien no se puede demostrar que la educación "saca” a los pobres de la pobreza, tampoco se puede demostrar que se puede prescindir de ella en el combate a la pobreza. La educación no resuelve todos los problemas, pero sin ella el cambio social para lograr una sociedad más justa será muy difícil.

Sin educación, las posibilidades de exigir el cumplimiento de los derechos sociales, económicos, culturales, civiles y políticos son mucho más estrechas; es por ello que la educación abre las puertas a otros derechos. Sin educación no hay ciudadanía posible y sin ciudadanía toda posibilidad de democracia es una quimera.

"La participación de los niños en los procesos de plantío y cosecha, cuidado de animales domésticos, desarrollo de actividades de pastoreo, entre otros. Son, en su conjunto, tareas necesarias para la subsistencia familiar y también actividades a través de las cuales los niños interiorizan pautas de comportamiento y repiten padrones y estereotipos laborales que se transmiten de generación en generación." (Del Alamo, Oscar 2003)

Trabajo no doméstico/no remunerado, donde se incluyen las labores vinculadas a la producción agrícola y ocupaciones afines, así como actividades que, sin estar directamente vinculadas a la agricultura, constituyen un elemento importante en las estrategias de auto-consumo y sobrevivencia familiar. Aran, siembran, echan salitre, de todo. Empiezan a trabajar de chiquititos. De más edad hacen trabajos más pesados. Como acá cada casa tiene su pedacito de terreno también hacen sembrado. Los niños en la casa muchas veces tienen que hacer el trabajo de la mamá, que sale a trabajar con su marido. Porque aquí también aran las mujeres. O se ocupan en el trabajo temporal. Entonces los niños tienen que quedarse en la casa a cargo de sus hermanitos más chicos, hacer la comida y en fin son verdaderas dueñas de casa, sobre todo las niñitas. (Canda B; Santiago W, 2009)

Una de las estrategias familiares que permite a los miembros adultos obtener ingresos es el trabajo infantil, para el caso de familias de escasos recursos, una vez que se produce el desempleo de uno de los padres o de algún adulto, se produce la incorporación de los niños a actividades productivas, que asumen las más variadas formas, mendicidad, venta callejera de dulces y calendarios, porteo en supermercados y ferias libres y otras por el período en el cual los adultos están desempleados. (Anthony P, 1980)

\section{ConCLusión}

A modo de conclusión se puede señalar que la participación de los niños en actividades laborales en ámbitos intra y extra familiares es vista como una actividad si no normal, a lo menos deseable por parte de quien forma parte de una unidad socioeconómica en situación de pobreza. El trabajo entonces no es la suma de acciones individuales y autónomas, el trabajo es social, es reconocido y reconocible, el trabajo tiene significado" para que el trabajo signifique algo... debe tener significado para la persona que lo lleva a cabo, en el mundo occidental trabajar significa madurez.

Muchos trabajos empíricos encuentran que los cambios en el ingreso tienen efectos marginales muy pequeños sobre las decisiones de desertar y trabajar aunque los trabajos empíricos no son concluyentes al 
respecto. El salario mínimo no se tendrá en cuenta en este trabajo; esta deficiencia social donde el factor principal está en la POBREZA MENTAL, donde nuestros campesinos se formulan en su corazonada que para salir de la penuria se necesita trabajar fuertemente teniendo una referencia en un plano practico como es el empleo infiltrándolo a nuestra niñez, dándole un segundo plano el resto de las necesidades que todo ser humano necesita incluyendo la educación.

En consecuencia, se necesita la mayor batalla que es eliminar en su percepción ideológica este conflicto que el estudio es menor que el trabajo o viceversa. Coexistiendo una realidad que pocos han considerado que ambos han persistido junto ya que los dos han estado de la mano para logra el éxito en la vida y así salir de ambas pobreza; mental y material. Estos dos fundamentos no están divorciados ni que depende del uno con el otro. Por lo tanto, el trabajo y la educación son las formulas perfecta para cohabitar en este mundo con muchos problemas sociales - económicos, que para salir de la pobreza se necesita que cada persona debe unificarla y aplicarla en cada faceta de su vida. También es importante tener presente que el aprovechamiento del niño en la escuela depende mucho de la calidad de ésta. El papel de la educación ha sido el eje fundamental para contribuir al cambio social y económico en el mundo.

Es por ello que Nicaragua, un país formada por ciudadanos de luchas en la cuales destacan su libertad de conciencia y decisiones firmes en la muchas generaciones que la conforma con una visión diferente creciendo y desarrollándose en el buen vivir, las buenas costumbres de fe, paz y cuidado, en la que miembros de cada familias tenga a diario felicidad, sustento en sus mesas; que nuestros niños, niñas, adolescentes, jóvenes, mujeres y hombres se preparen en los diversos programas educativos que conlleva a las escuela, en seguida a las universidad o carreras técnicas para formar los profesionales del hoy y del mañana que demanda nuestra sociedad, para conseguir alcanzar cada ser humano el desarrollo sostenible que se aspiran. De tal manera, el objetivo de este ensayo es tener una idea previa acerca del trabajo infant il en la zona rural, por lo que en lo cual se ha observado en que la familia del campo no les permita ver la educación como las preparación del cuerpo, alma y espíritu; en la que puede dar un giro en la formación de buenos líderes y dirigentes futuros de este país.

\section{Referencia Bibliográfica}

Akabayashi, H., \& Psacharopoulos, G. (1999). The trade - off between child labour and human capital formation: A Tanzanian case study. The Journal of Development Studies, 35(5), 120-140.

Alarcón Glasinovich, Walter (2004.). ¿Por qué erradicar el trabajo infantil? Revista Electrónica DHIAL, Barcelona: Instituto Internacional de Gobernabilidad, n. 33, 3 sept. 2002. Disponíve l em: . Acceso em: 20 mayo

Anker, R. (2000). La economía del trabajo infantil Criterios para su medición. Revista Internacional del Trabajo, 119(3), 283-309.

Anthony, P (1980) " El trabajo humano y la pérdida del significado", en: Revista Internacional de Ciencias Sociales, Vol.XXXII, No3.

Arenas-Monreal, L., Paulo-Maya, A., \& López-González, H. E. (1999). Educación popular y nutric ión infantil: experiencia de trabajo con mujeres en una zona rural de México. Rev. Saúde Pública, 33(2), 113-121.

Ávila-Curiel, A., Shamah Levy, T. E. R. E. S. A., Galindo Gómez, C. A. R. L. O. S., Rodríguez Hernández, G. E. R. A. R. D. O., \& Barragán Heredia, L. M. (1998). La desnutrición infant il en el medio rural mexicano. Salud PublicaMex, 40.

Ávila, A. S. (2007). Trabajo infantil e inasistencia escolar. Rev. Bras. Educ, 12(34), 68-80.

Briceño Ayala, L., \& Pinzón Rondón, Á. M. (2004). Efectos del trabajo infantil en la salud del menor trabajador. Revista de Salud Pública, 6(3), 270-288.

Canda, B., \& Santiago, W. (2009). Determinantes del Trabajo Infantil y Adolescente en Nicaragua. https:// repositorio.uchile.cl/handle/2250/102130 
DEL ÁLAMO, Oscar (2003.). Los niños del Perú. Cómo afrontar el trabajo infantil desde la cooperación al desarrollo. Magazine DHIAL, Barcelona: Instituto Internacional de Gobernabilidad, n. 14, p. 1-3, 2002. Disponible en: http://www.iigov.org/dhial/dh14/todo.htm>. Acessoem:

Esteve, J. M. (2003). La tercera revolución educativa. La educación en la sociedad del conocimiento,n6.7 262. https:// dialnet.unirioja.es/servlet/articulo?codigo $=1049534$

Ferrer, A. T. (2010). Educación obligatoria, asistencia escolar y trabajo infantil en España en el primer tercio del siglo XX. Historia de la Educación, 6.

Gajardo, M., \& de Andraca, A. M. (1988). Trabajo infantil y escuela. Santiago: FLACSO.

Garrabou, R. (2000). Lescola d'Enginyers Industrials de Barcelona (1851-1936). Tècnics i tecnologia en el desenvolupament de la Catalunya contemporània.

GATTINO, Silvia y Nora Aquino.(1999)Las familias de la nueva pobreza.Buenos Aires: Espacio Editorial.

Khoudour Castéras, D. (2007). ¿ Por qué emigran los colombianos? Un análisis departamental basado en el Censo de 2005. Revista de economía institucional, 9(16), 255-271.

Klein, E. (1993). El mundo del trabajo rural. Nueva sociedad, 124, 72-81.

Latorre Antonio (2003).La investigación acción. Conocer y cambiar la práctica educativa. 1ra ed. Editorial Graó. 5-138

Liebel, Manfred (1996) Capítulo, I. INFANCIA Y TRABAJO Para una mejor comprensión de los niños y niñas trabajadores en diferentes culturas y continentes Índice. IFejant

Liebel, Manfred (1996): somos NATRAS; Testimonios de Niños Trabajadores de Nicaragua Managua: ed. nueva Nicaragua.

Llop, J. M. B. (2000). Antes de nacer sabíamos trabajar: absentismo escolar y trabajo infantil en el Madrid rural del primer tercio del siglo XX. Historia agraria: Revista de agricultura e historia rural, (20), 169-194.

Maureira Estrada Fernando (2000) Trabajo Infantil: Algunas consideraciones desde la antropología.Revista Austral de ciencias sociales. n6, 113-123. http://revistas.uach.cl/pdf/racs/n6/art09.pdf

Salazar, M. C. (1995). La significación social del trabajo infantíl y júvenil en America latina y el Caribe. Trabajo Infantil. ¿Ser o no ser?, 63-88.

Salazar, M. C. (1996). El trabajo infantil en América Latina. Desarrollo y Cooperación, (1), 16.

Sapelli, C., \&Torche, A. (2004). Deserción escolar y trabajo juvenil:̨ dos caras de una misma decisión?.Cuadernos de economía, 41(123), 173-198.

Stewart, M. A. (2007). A Través de Nuestros Ojos: Un Estudio Fotográfico de Niños Trabajadores en Cochabamba, Bolivia. ISP Collection, 180.

Garrabou. R (2000). La organización del trabajo en el mundo rural y sus evoluciones históricas: Época contemporánea. Historia agraria: Revista de agricultura e historia rural, (20), 25-38.

Ray, R., \& Lancaster, G. (2005). Efectos del trabajo infantil en la escolaridad. Estudio plurinacio nal. Revista Internacional del Trabajo, 124(2), 209-232. 